\title{
Conductivity type conversion in p-CdZnTe under pulsed laser irradiation
}

\author{
V.V. Tetyorkin, A.V. Sukach, N.M. Krolevec \\ V. Lashkaryov Institute of Semiconductor Physics, NAS of Ukraine, Ukraine \\ Phone: 38 (044) 525-5461; e-mail: teterkin@isp.kiev.ua
}

\begin{abstract}
Laser-induced surface modification is investigated in $\mathrm{p}-\mathrm{Cd}_{0.9} \mathrm{Zn}_{0.1}$ Te under irradiation with nanosecond pulses of YAG:Nd laser by using the power density $5 \mathrm{MW} / \mathrm{cm}^{2}$. Conductivity type conversion of the near-surface region with the thickness of several micrometers is observed. The surface-barrier structures were prepared by electrolytic deposition of Au onto the irradiated surface. The spectral dependences of the photovoltaic response has been explained by formation of the graded band gap in the irradiated region. The direct current conductivity has been proved to be determined by the dislocation network.
\end{abstract}

Keywords: CdZnTe, laser irradiation, dislocations, conductivity type conversion.

Manuscript received 07.02.14; revised version received 08.07.14; accepted for publication 16.09.14; published online 30.09.14.

\section{Introduction}

Surface modification in semiconductors under pulsed laser radiation is increasingly used in opto- and nanoelectronics. By changing the laser processing conditions (intensity, duration and repetition rate of the laser pulse, parameters of the surface scannning) the surface structures with characteristic lateral and vertical dimensions ranged from nano- to micrometers can be obtained [1]. With respect to $\mathrm{CdTe}$ and $\mathrm{Cd}_{0.9} \mathrm{Zn}_{0.1} \mathrm{Te}$ solid solution, it can be of practical significance, since these materials are widely used in solar energy and nuclear spectrometry. The majority of the investigations of surface modification by using laser irradiation in these materials have concentrated on surface morphology and composition changes. Also, some physical properties were investigated in the irradiated CdZnTe [2-7]. The aim of this work is to study electrical and photoelectrical properties of barrier structures prepared on the lasermodified surface of $\mathrm{Cd}_{0.9} \mathrm{ZnTe}$ single crystals.

\section{Experimental details}

The samples used for the investigations were made of monocrystalline semi-insulating $\mathrm{Cd}_{0.9} \mathrm{Zn}_{0.1} \mathrm{Te}$ with the resistivity $(1 \ldots 2) \cdot 10^{10} \Omega \cdot \mathrm{cm}$ at room temperature. Preparation of samples included mechanical grinding and polishing followed by chemical-mechanical polishing in bromine-methanol etchant and removing excess tellurium from the surface. After that, they were irradiated with the second harmonic $(\lambda=532 \mathrm{~nm})$ of a neodymium laser with the pulse duration $10 \mathrm{~ns}$, $5 \mathrm{MW} / \mathrm{cm}^{2}$ intensity and repetition frequency $10 \mathrm{~Hz}$. Laser radiation was focused on the sample surface $4 \times 8 \mathrm{~mm}$ in a spot with a diameter of $3 \mathrm{~mm}$. To modify the surface, approximately $10^{3}$ pulses were used at each point, i.e. the irradiation dose was approximately $50 \mathrm{~J} / \mathrm{cm}^{2}$. The surface scanning was performed with the step $20 \mu \mathrm{m}$. The surface-barrier structures were prepared using electrolytic deposition of gold at room temperature and thermal evaporation of indium onto the samples 
heated up to $120^{\circ} \mathrm{C}$. It is known that these metals are widely used for preparation rectifying and ohmic contacts to CdTe of $p$ - and $n$ - type conductivity. The contact pad area was $\sim 10^{-2} \mathrm{~cm}^{2}$. The contacts were illuminated with monochromatic radiation to measure the spectral dependences of the photovoltaic response and with concentrated radiation of the $250-\mathrm{W}$ silicahalogen lamp to determine the open-circuit voltage.

\section{Experimental data and discussion}

Reliable determination of the conductivity type in semiinsulating CdTe and CdZnTe is not a trivial task, especially in the case of $p$-type conductivity. The wellknown methods based on Hall effect and thermoelectric current ("hot probe") measurements do not provide unambiguous results, which is caused by formation of the $n$-type conductivity layer at the surface of samples [8]. Moreover, correct measurements of the Hall effect require formation of the ohmic contact, which is problematic in $p$-type material. Because of a low concentration and low mobility of holes in these materials, their product is small, causing an ambiguous interpretation of measuring results [8].

Taking it into account, to determine the conductivity type at the surface the photovoltaic response in Schottky contacts was measured. When illuminated Schottky contacts generate nonequilibrium electron-hole pairs that are separated by the internal electric field of a potential barrier. Consequently, the measured signal registered on the contact pad is determined by nonequilibrium minority carriers [9]. In Schottky contacts made of $n$-type semiconductor, the detected signal of positive polarity is observed (photovoltage or photocurrent in open and short circuit mode, respectively). Note also that Fowler photoemission from metal to semiconductor generates the signal of the same polarity. Also, the important advantage of this method is its locality.

The pronounced photoresponse was observed in $\mathrm{Au}$ and In contacts deposited on the laser-modified surface and on the non-irradiated surface, respectively. The maximum value of the open circuit voltage was 110 down to $60 \mathrm{mV}$ in $\mathrm{Au}$ and In contacts, respectively. It should be pointed out that the measured signals had the opposite polarity in these contacts. In $\mathrm{Au}$ contacts, the positive polarity of the measured signal is caused by accumulation of holes at the contact pad. It proves the $n$ type conductivity of the laser-modified surface layer. The opposite sign of the signal in In contacts indicates the $p$-type conductivity of the non-irradiated surface. The Au contact deposited on the non-irradiated surface exhibited quasi-ohmic behavior (the open-circuit voltage of several millivolts was measured). The same is true for the In contact deposited on the laser-modified surface.

Formation of rectifying and ohmic contacts was also proved by measuring current-voltage characteristics (Fig. 1). Note that the direct current branches correspond to positive polarity of the applied bias on the Au contact and negative one on the In contact, respectively. The rectification factor measured at $U= \pm 10 \mathrm{~V}$ was $\sim 200$ and $\sim 5$ for these contacts. Smoother character of $I-U$ dependence measured on the initial surface is due to large series resistance, as compared to the structure on the modified surface $\left(2 \times 10^{9}\right.$ and $1.4 \times 10^{7} \Omega$, respectively, at the forward bias $50 \mathrm{~V}$ ). Fig. 2 shows the temperature dependence of the direct current in the barrier structure on MP. At $T<250 \mathrm{~K}$, the current weakly depends on temperature and can be approximated by a power law $I \sim$ $T^{1.6}$, and at $T>250 \mathrm{~K}$, it increases exponentially with temperature. The forward bias was chosen to eliminate the effect of barrier transport mechanisms on the measured current. Note that in $\operatorname{In} / p$-CdTe contacts prepared on the non-irradiated surface the temperature dependence of the dark current had an activation character caused by excitation of carriers from deep levels.

The spectral dependences of the photovoltaic response measured in the short-circuit current mode are shown in Fig. 3. The selective photoresponse with a maximum at $0.79 \mu \mathrm{m}$, which corresponds to the energy gap of CdZnTe, is due to surface recombination. The main feature of the photoresponse in the barrier structure is appearance of a wide spectral band within the range $0.55 \ldots 0.63 \mu \mathrm{m}$. Its origin may be caused by several reasons.

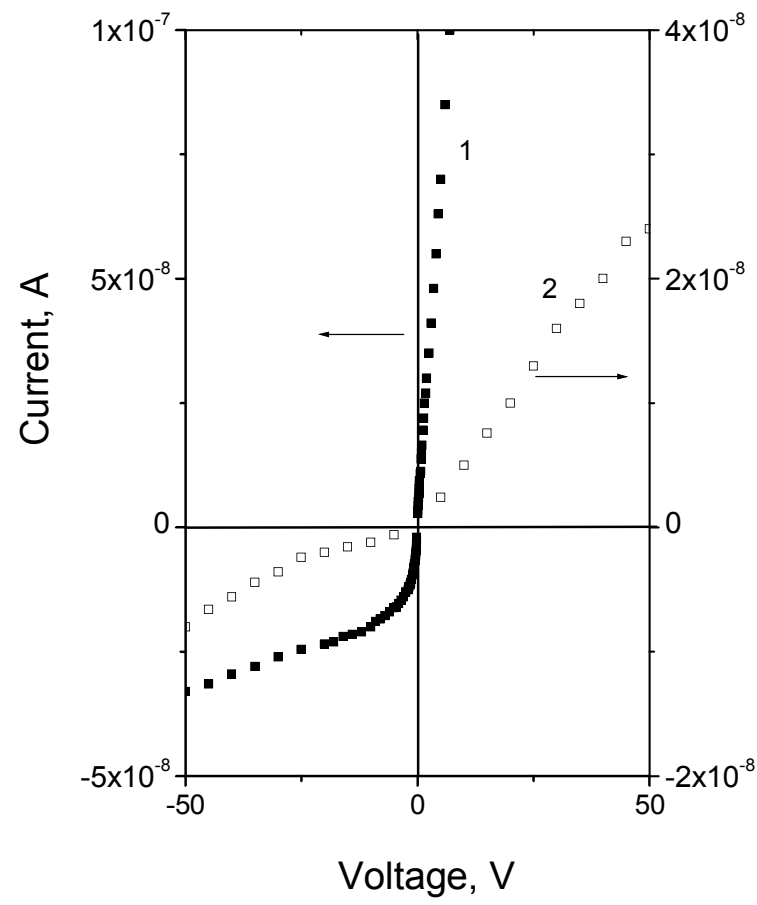

Fig. 1. Current-voltage characteristics in $\mathrm{Au} / n-\mathrm{Cd}_{0.9} \mathrm{Zn}_{0.1} \mathrm{Te}$ (close dots) and $\mathrm{In} / p-\mathrm{Cd}_{0.9} \mathrm{Zn}_{0.1} \mathrm{Te}$ (open dots) contacts. 


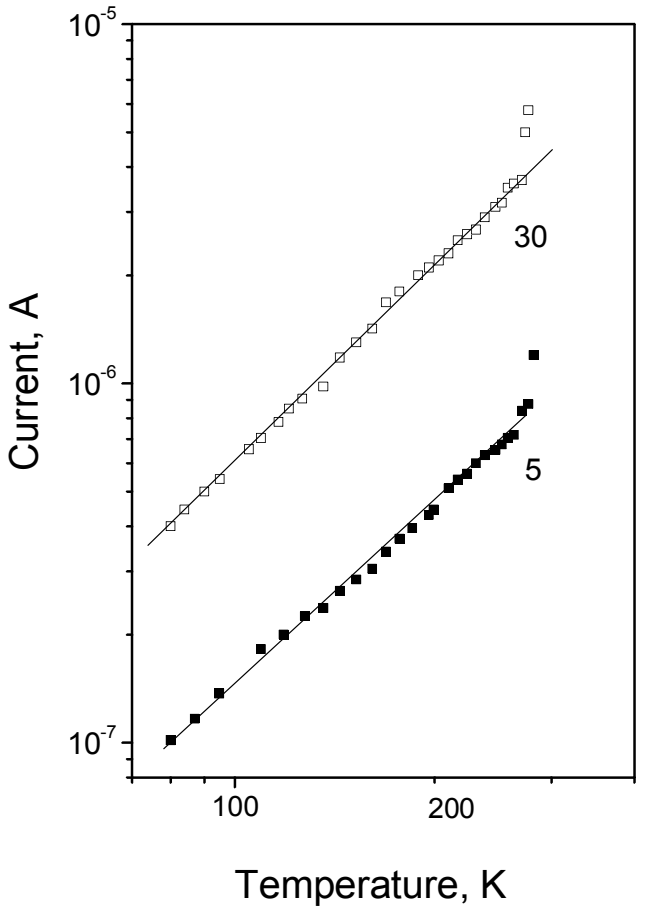

Fig. 2. Temperature dependences of the current-voltage characteristics in $\mathrm{Au} / n-\mathrm{Cd}_{0.9} \mathrm{Zn}_{0.1} \mathrm{Te}$. Numbers at the curves are values of the forward bias voltage in volts.

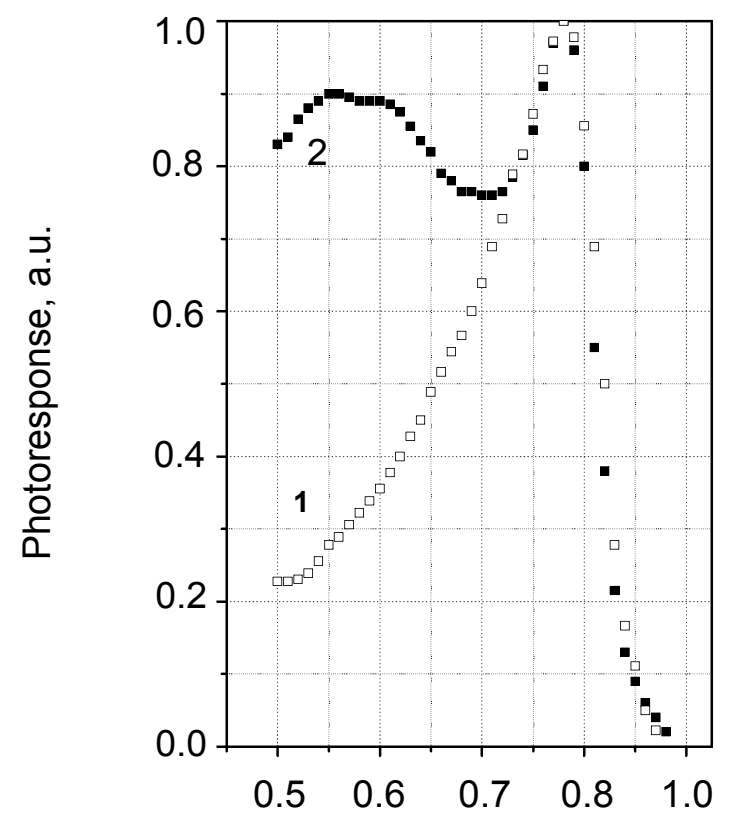

Wavelength, $\mu \mathrm{m}$

Fig. 3. Photosensitivity spectra in contacts on the nonirradiated (1) and irradiated (2) surfaces measured in the shortcircuit current mode at $T=295 \mathrm{~K}$.
Laser-induced modification of the surface in CdTe and CdZnTe was investigated in a number of studies using single and multiple irradiation of ruby and neodymium laser [2-7]. By using multiple irradiation, surface modification was carried out in the following order: emergence of excess tellurium, formation of dislocation networks and nanostructures $[3,4]$. The shape of nanostructures, their vertical and lateral dimensions as well as the surface density were determined by the laser fluence. For single pulse irradiation, the melting threshold for CdTe is within $35 \ldots 50 \mathrm{~mJ} / \mathrm{cm}^{2}$ [5]. Since the thermal and optical parameters of cadmium telluride and solid solutions $\mathrm{Cd}_{0.9} \mathrm{Zn}_{0.1}$ Te are close, the possibility of melting the surface in the investigated samples cannot be excluded. It should be also noted that under multiple irradiation the threshold melting point can be achieved at lower intensities due to cumulative mechanism of defect generation [4]. According to modern concepts, the laserinduced nanostructures in semiconductors are caused by instabilities of different nature [1]. In application to CdTe, models of defect-deformation and plasmadeformation instabilities were developed $[1,6]$.

Because under irradiation with multiple pulses the surface layer of $n$-type conductivity is formed, the photovoltaic response can be determined by the Schottky barrier at the surface, as well as the $p$ - $n$ junction under the surface. The photoresponse generated by these barriers photoresponse should have the opposite sign. The lack of the sign inversion in Fig. 3 may indicate that the depth of the $p-n$ junction is sufficiently large (longer than the diffusion length of non-equilibrium holes), whereby it does not affect the measured photoresponse. Note also that the long-wave edge of the photoresponse $\lambda_{c} \cong$ $0.81 \mu \mathrm{m}$ corresponds to the absorption depth of the order of $1.8 \mu \mathrm{m}$, as the the absorption coefficient at this wavelength is $5.5 \cdot 10^{3} \mathrm{~cm}^{-1}$ [10]. This value can be interpreted as the lower limit of the $p$ - $n$ depth conversion. The above facts indicate that the depth of the surface modification exceeds significantly the depth of laser radiation absorption $\sim 0.1 \mu \mathrm{m}$, as well as the depth of heat penetration $\sim 0.2 \mu \mathrm{m}$ determined by thermal diffusivity [5]. The performed estimations are in accordance with the values $1.5 \ldots 3 \mu \mathrm{m}$ obtained earlier for the thickness of the laser-modified surface layer in CdTe [11, 12]. A possible explanation for these values can be impact of the shockwave produced by the pulsed laser irradiation on clusters of point defects and dislocations [13].

The short-wave peak in the spectral dependence of the photoresponse in Fig. 3 can be explained by the presence of the graded-gap region in the modified surface layer. Note that this peak correlates with the spectral position of the photoluminescence band $1.85 \ldots 1.90 \mathrm{eV}$ found in $\mathrm{Cd}_{0.9} \mathrm{Zn}_{0.1} \mathrm{Te}$ irradiated by neodimium laser with $9.0 \ldots 12.0-\mathrm{MW} / \mathrm{cm}^{2}$ intensity, which has been explained by formation of a nanostructured surface layer [4]. Several reasons were also indicated for graded-gap region formation, namely: 
redistribution of $\mathrm{Cd}$ and $\mathrm{Zn}$ and strain effects at the undersurface region $[1,4,14]$.

The power dependence of the dark current on temperature can indicate the dislocation mechanism of its flow. Note that formation of the dislocation network in CdTe were observed experimentally at doses exceeding $0.8 \mathrm{~J} / \mathrm{cm}^{2}[4,7,12]$. Similar temperature dependences of the dark current were observed in plastically strained $n$-Ge within the range $0.01 \ldots 40 \mathrm{~K}$. It was explained by the model of percolation conductivity through the dislocation network [14]. In this case, the power dependence of the dark current is observed at a relatively high temperature due to a lower concentration of charge carriers $\left(\sim 10^{7}\right.$ and $\sim 10^{12} \mathrm{~cm}^{-3}$ at ambient temperature, respectively) as well as lower carrier mobility in $\mathrm{Cd}_{0.9} \mathrm{Zn}_{0.1} \mathrm{Te}$ as compared to $n$ Ge. The observed conversion of the conductivity type is not trivial, since it is generally accepted that laser irradiation results in formation of cadmium vacancies (stoichiometry violation) [7, 15]. A possible reason for the observed conversion is generation of a large amount of dislocations and interstitial cadmium.

\section{Conclusions}

Pulsed laser irradiation of $p-\mathrm{Cd}_{0.9} \mathrm{Zn}_{0.1} \mathrm{Te}$ results in formation of the surface layer with $n$-type conductivity. Conversion of the conductivity type occurs at the micrometer depth. The photovoltaic spectra in the surfacebarrier structures prepared on the laser-modified surface has been explained by appearance of the graded-gap region. The temperature dependence of the dark current seems to be determined by the dislocation network.

\section{Acknowledgments}

The authors would like to thank Prof. A. Medvid' for providing samples of $\mathrm{CdZnTe}$ and helpful discussion.

\section{References}

1. N. Mirzoev, V.Ya. Panchenko, L.A. Shelepin, Laser control processes in solids // Phys. Usp. 39(1), p. 1-29 (1996).

2. A. Medvid', P. Onufrijevs, A. Mychko // Nanoscale Res. Lett. 6, p. 582 (2011).

3. A. Baidullaeva, A.I. Vlasenko, L.F. Cuzan, O.S. Litvin, P.E. Mozol', The formation of nanodimensional structures on the surface of $p$ CdTe crystals exposed to a single radiation pulse from a ruby laser // Semicond. (Springer), 39(9), p. 1028-1031 (2005).
4. A. Baidullaeva, M.B. Bulakh, A.I. Vlasenko, A.V. Lomovtsev, P.E. Mozol', The evolution of surface structures in $p$-CdTe crystals under pulsed laser irradiation // Semicond. (Springer), 38(1), p. 23-26 (2004).

5. V.A. Gnatyuk, T. Aoki, O.S. Gorodnychenko, Y. Hatanaka // Appl. Phys. Lett. 83(18), p. 3704-3706 (2003).

6. V.I. Emel'yanov, A. Baidullaeva, A.I. Vlasenko, L.F. Kuzan, O.S. Lytvyn, P.E. Mozol', Plasmadeformation mechanism of nanocluster array formation on CdTe crystal surface under the action of a single laser pulse // Techn. Phys. Lett. 32(8), p. $732-734$ (2006).

7. I.L. Shul'pina, N.K. Zelenina, O.A. Matveev, Heat effect of pulsed laser radiation on the real structure of CdTe single crystals // Phys. Solid State, 42(3), p. 561-565 (2000).

8. M. Prokesch and C. Szeles, Accurate measurement of electrical bulk resistivity and surface leakage of CdZnTe radiation detector crystals // J. Appl. Phys., 100, 014503 (2006).

9. A.G. Milnes and D.L. Feucht, Heterojunctions and Metal-Semiconductor Junction. Academic Press, 1972.

10. S.S. Ou, O.M. Stafsudd, B.M. Basol, Optical properties of electrochemically deposited CdTe films // J. Appl. Phys. 55(10), p. 3769-3772 (1989).

11. V.N. Babencov, A. Baidullaeva, A.I. Vlasenko et al., Mechanism of formation of disrupted layer in p-CdTe under irradiation of nanosecond laser pulses // Sov. Phys. Semicond., 27(10), p. 16181623 (1993).

12. I.L. Shul'pina, V.V. Ratnikov, O.A. Matveev, Xray diffraction study of changes in the $\mathrm{CdTe}$ monocrystal real structure induced by laser radiation // Phys. Solid State, 43(3), p. 579-582 (2001).

13. A. Baidullaeva, A.I. Vlasenko, B.L. Gorkovenko, A.V. Lomovtsev, P.E. Mozol', Variation in the defect structure of $p$-CdTe single crystals at the passage of the laser shock wave // Semicond. (Springer), 34(4), p. 429-432 (2000).

14. S. A. Shevchenko, Influence of annealing on the dislocation-related electrical conductivity of germanium // Semicond. (Springer), 34(5), p. 527533 (2000).

15. 15. P.D. Brewer, J.J. Zinck, and G.L. Olson, Reversible modification of CdTe surface composition by excimer laser irradiation // Appl. Phys. Lett. 57(24), p. 2526-2528 (1990). 